\title{
Insights into the Origin of the Galaxy Mass-Metallicity Relation
}

\author{
Henry Lee ${ }^{1}$, Eric F. Bell ${ }^{2}$, and Rachel S. Somerville ${ }^{2,3}$ \\ ${ }^{1}$ Gemini Observatory, AURA Chile \\ Colina El Pino s/n, La Serena, Chile \\ email: hlee@gemini.edu \\ ${ }^{2}$ Max-Planck-Institut für Astronomie \\ Königstuhl 17, D-69117 Heidelberg, Germany \\ email: bell@mpia.de \\ ${ }^{3}$ Present address: Space Telescope Science Institute \\ 3700 San Martin Drive, Baltimore, MD 21218 USA \\ email: somer@stsci.edu
}

\begin{abstract}
We examine mass-metallicity relations for nearby $(D<2 \mathrm{Mpc})$ gas-rich and gas-poor dwarf galaxies. We derived stellar and baryonic masses using photometric data and used average stellar iron abundances as the metallicity indicator. With the inclusion of available data for massive galaxies, we find a continuous mass-metallicity relation for galaxies spanning nine orders of magnitude in mass, and that the mass-metallicity relations are the same for both gas-rich and gas-poor dwarf galaxies. We derive stellar effective yields from the stellar abundances, finding that gas-poor dwarf galaxies form a single sequence with mass, whereas gas-rich dwarf galaxies have higher yields at comparable mass. Simple chemical evolution models show that a massdependent star-formation efficiency can simultaneously account for the correlations between metallicity, gas fraction, and stellar effective yield with mass. In agreement with recent and independent results, we conclude that a key driver of the mass-metallicity relation is the variation of star-formation efficiency with galaxy mass, modulated by galaxy mass-dependent outflows and/or stellar IMF variations, and coupled with environmental gas-removal processes.
\end{abstract}

Keywords. galaxies: abundances, galaxies: dwarf, galaxies: evolution, galaxies: stellar content, Local Group

\section{Introduction}

It has long been recognized that the relationship between the chemical abundances and the properties of galaxies is a key diagnostic of the physical processes that shape their evolution. The best-known of these relations is the luminosity-metallicity $(L-Z)$ relation, observed in the nearby universe across a large dynamic range in luminosity from dwarf to giant galaxies for both star-forming gas-rich types (e.g., Tremonti et al. 2004, Lee et al. 2006) and quiescent gas-poor types (e.g., Grebel et al. 2003, Gallazzi et al. 2005). Although the methods most frequently used to measure metallicity depend on whether galaxies have gas (i.e., nebular oxygen abundances in gas-rich systems, and stellar iron abundances in gas-poor systems), the sense of the $L-Z$ relations is the same: more luminous galaxies are more metal-rich.

Many different physical processes may contribute to the chemical evolution of galaxies; e.g., variations in star formation efficiency, infall of pristine gas diluting the more metal-rich interstellar medium of the galaxy, outflows driven by supernovae, ram-pressure stripping, metallicity-dependent supernova yields, systematic changes in the galaxyscale stellar initial mass function. Many of these physical processes are demonstrated to 
account for the observed $L-Z$ relations without the influence of other physical processes, all of which makes convergence towards a unique model of chemical evolution challenging.

\section{Mass-Metallicity $(M-Z)$ Relations}

We took a sample of 65 nearby $(D \leqslant 2 \mathrm{Mpc})$ dwarf galaxies, based upon the compilation in Grebel et al. (2003; GGH03) and the recent discovery of dwarfs in the Local Group; see Lee et al. (2008; LBS08) for a complete list of references. We included a sample of massive galaxies taken from the Sloan Digital Sky Survey Data Release 2 (SDSS DR2), using stellar metallicities from Gallazzi et al. (2005) and estimates of stellar and gas masses from Bell (2003). For the dwarf galaxies, the methods by which masses are derived and how stellar metallicities have been assigned (especially for gas-rich dwarfs) are discussed in much greater detail in LBS08.

The fits for mean stellar metallicity against stellar and baryonic mass are expressed as

$$
\begin{aligned}
& {[\mathrm{Fe} / \mathrm{H}]=(-3.74 \pm 0.16)+(0.354 \pm 0.025) \log \left(M_{*} / M_{\odot}\right)} \\
& {[\mathrm{Fe} / \mathrm{H}]=(-3.68 \pm 0.16)+(0.338 \pm 0.024) \log \left(M_{b} / M_{\odot}\right)}
\end{aligned}
$$

There is a clear correlation between stellar metallicity and stellar (or baryonic) mass. We
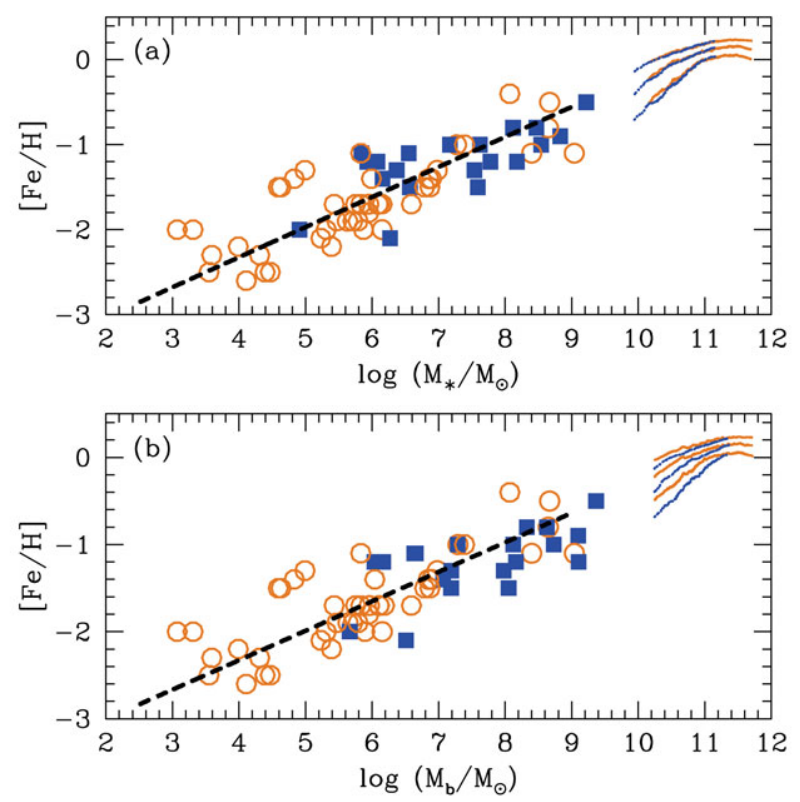

Figure 1. Mass-metallicity relations spanning $\sim 9$ dex in galaxy mass. Mean stellar iron abundance versus stellar mass (a) and baryonic mass (b). Open circles in orange denote nearby $(D \leqslant 2$ $\mathrm{Mpc}$ ) gas-poor dwarf galaxies. Filled squares in blue denote nearby gas-rich dwarf galaxies. The median metallicity and upper and lower quartiles as a function of stellar mass are also plotted for massive galaxies from the SDSS DR2. The high-mass sample is coded into gas-rich (blue) and gas-poor (orange) populations. The fits in Equation (2.1) and (2.2) are shown as dashed lines in each panel.

place the $L-Z$ relation on a more physical basis when this relation is expressed in terms of stellar or baryonic mass instead of galaxy luminosity. The mass-metallicity correlation has some scatter, but the scatter appears relatively constant at all masses $\leqslant 10^{9} M_{\odot}$. The offset found previously in $L-Z$ relations no longer appears, because we have accounted 
for various methods by which stellar iron abundances are obtained in dwarf galaxies with a larger mix of recent or present-day star formation (see LBS08).

\section{Stellar Effective Yield}

To place gas-rich galaxies and gas-poor galaxies on the same scale, we derive the stellar effective yield. By analogy to the gas effective yield (Pagel 1997), stellar effective yield is defined by

$$
\begin{aligned}
y_{\mathrm{eff}}^{*} & =\frac{Z_{\mathrm{Fe}} / Z_{\mathrm{Fe}, \odot}}{<z>}=\frac{10^{[\mathrm{Fe} / \mathrm{H}]}}{<z>}, \\
<z> & =1+\frac{\mu \ln \mu}{1-\mu}
\end{aligned}
$$

where $Z_{\mathrm{Fe}} / Z_{\mathrm{Fe}, \odot}$ is the iron mass fraction relative to solar, $[\mathrm{Fe} / \mathrm{H}]=\log (\mathrm{Fe} / \mathrm{H})-$ $\log (\mathrm{Fe} / \mathrm{H})_{\odot}$, and $\langle z\rangle$ is the mean metallicity of a stellar population with remaining cold-gas fraction $\mu$ if the galaxy evolves as a closed box with yield $y_{\text {eff }}^{*}$ (i.e., as $\mu \rightarrow 0$, $<z>\rightarrow 1$ and $\log y_{\mathrm{eff}}^{*} \rightarrow[\mathrm{Fe} / \mathrm{H}]$; see Smith 1985 and Pagel 1997), under the assumption that the true yield is constant as a function of time and metallicity. The stellar effective yield is the mass of metals produced per unit stellar mass formed, normalized so that solar metallicity is unity. In essence, $y_{\mathrm{eff}}^{*}$ is the metals yield required to place a given galaxy on a "closed box" chemical evolution track, and attempts to encapsulate the "potential" of the galaxy to form metals in the future.

We have plotted stellar effective yields derived from Equation (3.1) against stellar mass and baryonic mass in Fig. 2. For galaxies with no measured gas masses, we assume
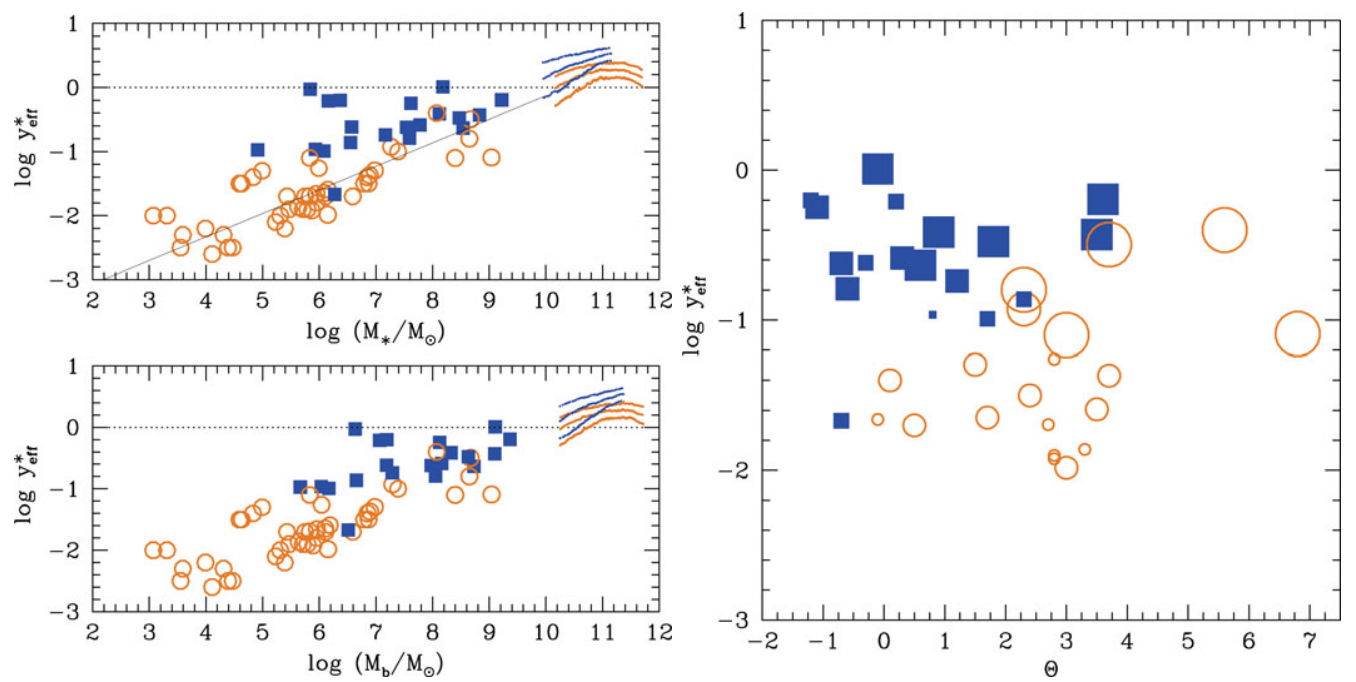

Figure 2. LEFT PANEL: Stellar effective yield versus (a) stellar mass and (b) baryonic mass. The dotted horizontal line in each panel represents $y_{\mathrm{eff}}^{*}=1$. Plot symbols and colors are the same as in Fig. 1. Uncertainties have not been plotted to improve clarity. Stellar effective yields correlate well with mass for gas-poor dwarfs; the fit to these dwarfs expressed in Equation (3.3) is shown as a thin solid line in panel (a). RIGHT PANEL: Stellar effective yield versus tidal index. Tidal indices are taken from Karachentsev et al. (2004). Plot symbols and colors are the same as in Fig. 1. The size of the plot symbols increases with stellar mass. That gas-rich dwarfs with higher stellar effective yields are found in relative isolation is very similar to the result that gas-rich dwarfs are found at large projected galactocentric distances, e.g., GGH03. 
their effective yields are equivalent to their stellar iron abundances. For the gas-poor dwarfs, there is a strong correlation between mass and effective yield, because it is almost equivalent to the $M-Z$ relation in the case for gas-poor galaxies. The resulting ordinary least-squares bisector fit to the gas-poor dwarfs is

$$
\log y_{\mathrm{eff}}^{*}=(-3.80 \pm 0.21)+(0.367 \pm 0.034) \log \left(M_{*} / M_{\odot}\right)
$$

where $M$ is either stellar or baryonic mass. Stellar effective yields for gas-rich dwarfs are higher than yields for gas-poor dwarfs at comparable mass, and are less sensitive to the dependence on stellar mass than gas-poor dwarfs.

We plot the stellar effective yield as a function of tidal index in Fig. 2, using available tidal indices from Karachentsev et al. (2004), where the tidal index of galaxy is a measure of proximity to a massive neighbor. Galaxies with negative tidal indices are isolated in the field, and those with positive tidal indices are found within higher-density (e.g., group) environments, where tidal interactions are more likely. We note that the tidal index is simply a measure of the present-day environment of a galaxy. The stellar effective yield is a function of environment: isolated (low tidal-index) gas-rich galaxies have higher stellar effective yields at a given stellar mass than group (high tidal-index) galaxies.

\section{Implications}

Taken together with the well-documented tendency for gas-poor dwarf galaxies to be found only in the vicinity of giant galaxies (as noted by GGH03), the present result lends support to the notion that most gas-poor dwarf galaxies have had their gas contents removed by (primarily hydrodynamical) stripping. This result helps to remove a key objection to a possible stripping origin for present-day gas-poor dwarf galaxies having evolved from gas-rich dwarf galaxies.

We explored the predictions of a number of simple models of chemical evolution, all of which reproduced the $M_{*}-Z$ relation, but for different physical reasons; see LBS08 for discussion. We found that while outflows alone can successfully imprint a correct $M-Z$ relation, such models are not capable of reproducing trends in stellar effective yields, cold-gas fractions, and star formation efficiencies. However, models in which the primary driver of the $M-Z$ correlation is a strong variation in star formation efficiency with mass are successful at simultaneously accounting for all of these observed trends. In Fig. 3, we show how the $M-Z$ relation is driven in equal measure by SFE variations and metal-enriched winds. There is freedom to choose model parameters in such a hybrid model, but from the scatter in the relations, it is not clear that it is a meaningful exercise to identify a "favorite" model. However, a combination of mass-dependent SFE and outflows (or potentially galaxy-scale IMF variations) can provide a framework from which one can meaningfully begin to interpret the behavior of nearby gas-rich galaxies.

We summarize with these three points. First, gas-rich and gas-poor dwarf galaxies have the same $M-Z$ relation, which when combined with a sample of more massive galaxies spans nine orders of magnitude in galaxy mass. Second, the $M-Z$ relation is defined primarily by the physics of gas-rich galaxies. Third, the primary driver of the $M-Z$ relation is the variation of star-formation efficiency with galaxy mass, modulated by galaxy mass-dependent outflows and/or variations in stellar IMF, and coupled with environmental gas-removal processes (e.g., stripping) at relatively late times. These results are 


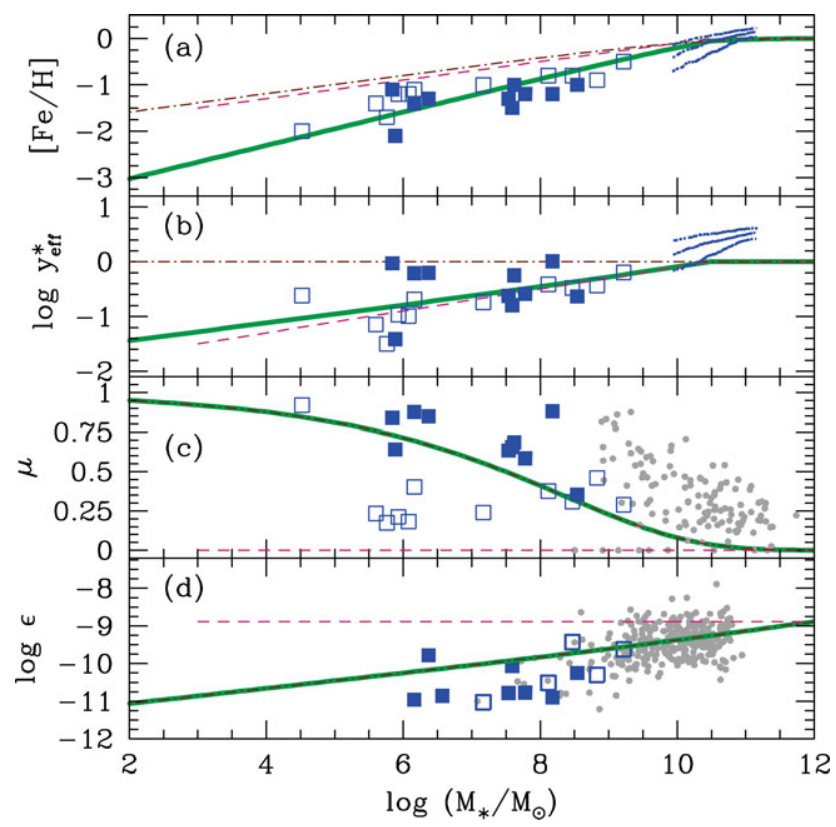

Figure 3. Trends in stellar metallicity (a), stellar effective yield (b), cold-gas fraction (c), and star-formation efficiency (d; SFE, in units of $\mathrm{yr}^{-1}$ ) with stellar mass for gas-rich galaxies. Blue squares represent the present sample of gas-rich dwarf galaxies; open (filled) symbols indicate less (more) isolated galaxies with tidal indices larger (smaller) than +0.7 . Small filled circles in grey in panel c show gas fractions for a sample of galaxies from Bell \& de Jong (2000), whereas the small filled circles in grey in panel d denote SFEs for a combined sample taken from Kennicutt (1998), van Zee (2001), and Bell (2003). Toy models are shown, where both the SFE and metal-enriched winds are a strong function of mass. The solid line denotes the "combined" model, the dash-dotted line shows the effect of mass-dependent SFE only, and the dashed line shows the contribution of the mass-dependent metal-enriched winds.

consistent with the conclusions independently drawn, for example, by Dalcanton (2007) and Ellison et al. (2008).

\section{Acknowledgements}

HL thanks the members of the conference LOC \& SOC for an excellent conference which was held at such a beautiful seaside venue. HL also acknowledges support from the Max-Planck Institute for Astronomy, the University of Minnesota, and Gemini Observatory.

\section{References}

Bell, E. F. 2003, ApJ, 586, 794

Bell, E. F. \& de Jong, R. S. 2000, MNRAS, 312, 497

Dalcanton, J. J. 2007, ApJ, 658, 941

Ellison, S. L., Patton, D. R., Simard, L., \& McConnachie, A. W. 2008, ApJ, 672, L107

Gallazzi, A., Charlot, S., Brinchmann, J., White, S. D. M., \& Tremonti, C. A. 2005, MNRAS, 362,41

Grebel, E. K., Gallagher, J. S., \& Harbeck, D. 2003, AJ, 125, 1926 (GGH03)

Karachentsev, I. D., Karachentseva, V. E., Huchtmeier, W. K., \& Kakarov, D. I. 2004, AJ, 127, 2031 
Kennicutt, R. C. 1998, ApJ, 498, 541

Lee, H., Skillman, E. D., Cannon, J. M., Jackson, D. C., Gehrz, R. D., Polomski, E. F., \& Woodward, C. E. 2006, ApJ, 647, 970

Lee, H., Bell, E. F., \& Somerville, R. S. 2008, ApJ, submitted (LBS08)

Pagel, B. E. J. 1997, Nucleosynthesis and the Chemical Evolution of Galaxies (Cambridge: CUP)

Tremonti, C. A., et al. 2004, ApJ, 613, 898

van Zee, L. 2001, AJ, 121, 2003 